\title{
Desempenho de cultivares de couve-chinesa em Cáceres-MT
}

\author{
Santino Seabra Júnior; Adelina S Pereira; Kelly L Araujo \\ UNEMAT, Av. São João s/n, Cavalhadas, 78200-000 Cáceres-MT; santinoseabra@hotmail.com; adelina_ad@hotmail.com; \\ kellylana_araujo@yahoo.com.br
}

\section{RESUMO}

A couve-chinesa é uma hortaliça que apresenta alto valor nutricional, sendo a escolha de genótipos adaptados às condições locais importante para o sucesso do seu cultivo. Assim, o presente trabalho teve como objetivo avaliar o desempenho de cultivares de couve-chinesa cultivadas em condições de altas temperaturas. $\mathrm{O}$ experimento foi conduzido na área experimental pertencente à Universidade do Estado de Mato Grosso, em Cáceres-MT, de 8 de abril a 23 de junho de 2011. O delineamento experimental utilizado foi de blocos ao acaso com cinco repetições, sendo avaliadas sete cultivares Granat, Michihilli, Kyoto, Seijin F1, Kinjitsu, AF 74 e AF 66. Avaliou-se o número de plantas pendoadas por parcela, produtividade, peso, diâmetro e altura da cabeça, comprimento do caule, altura da planta, número de folhas da saia e compacidade. Constatou-se que não houve diferença estatística, quanto à produtividade; entretanto, para a produção comercial a cultivar AF 66 apresentou melhor desempenho. A cultivar Seijin apresentou o maior diâmetro da cabeça $(13,2 \mathrm{~cm})$. Para altura das plantas os resultados foram inversamente proporcionais ao diâmetro da cabeça. A cultivar Michihilli apresentou a maior média para comprimento do caule $(17,4 \mathrm{~cm})$, visto que esta se encontrava com $9,1 \%$ das plantas florescida no dia da colheita. A cultivar AF 66 obteve-média de 4,3 para compacidade da cabeça. $\mathrm{O}$ número de folhas da saia foi em média 15 para a cultivar Kyoto. Entre as cultivares avaliadas, a AF 66 apresentou o melhor resultado com boa adaptação para a região de Cáceres-MT.

\begin{abstract}
Performance of Chinese cabbage cultivars in Cáceres, Brazil

The Chinese cabbage is a vegetable with high nutritional value. The choice for adapted genotypes to local conditions is important to provide success in its cultivation. Thereby, we evaluated the performance of Chinese cabbage cultivars grown under high temperature conditions. The experiment was carried out in the Universidade Estadual do Mato Grosso, in Cáceres, Mato Grosso state, Brazil, from April $8^{\text {th }}$ to June $23^{\text {rd }}$, 2011. The experimental design was randomized blocks with five replications. Seven cultivars were evaluated (Granat, Michihilli, Kyoto, Seijin F1 Kinjitsu, AF 74 and AF 66). We evaluated the number of bolted plants, productivity (PT) (t/ha), weight of head (g/plant), head diameter $(\mathrm{cm})$, head height $(\mathrm{cm})$, stem length $(\mathrm{cm})$, plant height $(\mathrm{cm})$, number of leaves of the skirt, and compactness. We found no statistical difference regarding productivity. AF 66 showed a better performance for head production. Seijin presented the highest head diameter $(13.2 \mathrm{~cm})$. Plant height results were inversely proportional to the head diameter. Cultivar Michihilli had the highest average value for stem length $(17.4 \mathrm{~cm})$, considereing $9.1 \%$ of plants flowered on the day of harvest. For head compactness we obtained a score of 4.3 for the cultivar AF 66. 'Kyoto' averaged 15 discarded leaves regarding the number of leaves of the skirt. Among the studied cultivars, AF 66 presented the best result, also showing good adaptation to the hot climate of Caceres.
\end{abstract}

Keywords: Brassica pekinensis, high temperatures, production.

Palavras-chaves: Brassica pekinensis, altas temperaturas, produção.

(Recebido para publicação em 23 de setembro de 2013; aceito em 27 de agosto de 2014) (Received on September 23, 2013; accepted on August 27, 2014)

\begin{abstract}
A couve-chinesa (Brassica pekinensis) é cultivada na China há mais de 1500 anos (Maroto-Borrego, 1995). No Brasil o consumo se dá principalmente devido à culinária nipônica, sendo erroneamente conhecida como acelga. A demanda de consumo vem aumentando devido ao seu alto valor nutricional, sendo excelente fonte de cálcio, potássio, vitaminas $\mathrm{A}$ e $\mathrm{C}$ e de ácido fólico (Ito et al., 2006), além de apresentar alto teor de fibras, imprescindíveis para a boa digestão, bem como na prevenção de certos tipos de câncer (Filgueira, 2008).

A couve-chinesa apresenta folhas de coloração verde-clara, oblongas cris-
\end{abstract}

padas, onduladas nas margens, pilosas, com nervura central carnosa e grossa de coloração branca, as folhas espessas se fecham formando uma cabeça compacta, globular alongada e com comprimento de 30 a $40 \mathrm{~cm}$ (Filgueira, 2008).

Essa espécie possui elevada produtividade (Ferreira et al., 2002), principalmente quando cultivada sob condições de outono-inverno, pois seu desenvolvimento é favorecido por temperaturas amenas. A passagem do estádio vegetativo para o reprodutivo é induzida por temperaturas baixas, sendo o seu florescimento inibido por altas temperaturas (Bernier \& Périlleux,
2004).

A escolha de genótipos adaptados às condições locais é decisiva para o sucesso do cultivo da couve-chinesa. Recomendações de cultivares têm sido realizadas por empresas produtoras de sementes para diversas regiões produtoras; porém, há grande demanda de resultados de pesquisas para condições tropicais, principalmente, em locais de altas temperaturas, como é o caso da região de Cáceres-MT. Esta região apresenta clima tropical com registro de médias anuais de temperatura de $31,5^{\circ} \mathrm{C}$ e mínima média de $20,1^{\circ} \mathrm{C}$, podendo ocorrer temperaturas de 2 a $41^{\circ} \mathrm{C}$ (Neves 
et al., 2011).

Diante disto, tornou-se necessária a realização deste estudo, buscando contribuir com os produtores da região, na avaliação do desempenho de cultivares de couve-chinesa em condições de altas temperaturas, visando identificar genótipos com maior produtividade.

\section{MATERIAL E MÉTODOS}

O experimento foi conduzido em campo experimental da Universidade do Estado de Mato Grosso, localizada no município de Cáceres-MT (1604'33’S, 57³9'10'O, altitude 118 m). A região apresenta clima tropical de altitude, inverno seco e chuvas no verão. No período de cultivo, de 08 de abril a 23 de junho de 2011 as médias das temperaturas máxima, média e mínima foram de $32,2,27,1$ e $23,5^{\circ} \mathrm{C}$, respectivamente (INMET, 2014).

O solo da área é classificado como Plintossolo Pétrico Concrecionário Distrófico (Embrapa, 2006).

Foram avaliadas sete cultivares de couve-chinesa [Granat $\left(\right.$ Hortec $^{\circledR}$ ), Michihilli $\left(\right.$ Feltrin $^{\circledR}$ ), Kyoto ${ }^{0}$ 3, Seijin F1, Kinjitsu $\left(\right.$ Topseed $^{\circledR}$ ), AF 74 e AF $66\left(\right.$ Sakata $\left.^{\circledR}\right)$ ]. O delineamento experimental foi em blocos ao acaso com cinco repetições, com 12 plantas por parcela, considerando-se úteis as seis plantas centrais. $\mathrm{O}$ cultivo foi realizado em canteiros de 9,0x1,2 m contendo três fileiras de plantas.

A adubação das plantas seguiu as recomendações de Trani et al (1997) para couve-manteiga e mostarda. Foi aplicado $40 \mathrm{t} /$ ha de esterco de curral curtido. Para a adubação mineral, foram aplicados $60 \mathrm{~kg} /$ ha de N, $200 \mathrm{~kg} / \mathrm{ha}$ de $\mathrm{P}_{2} \mathrm{O}_{5}$ e $120 \mathrm{~kg} /$ ha de $\mathrm{K}_{2} \mathrm{O}$, utilizando como fonte desses nutrientes o formulado 4-14-8 e o superfosfato simples $\left(18 \% \mathrm{P}_{2} \mathrm{O}_{5}\right) . \mathrm{Na}$ adubação mineral de cobertura aplicou-se $165 \mathrm{~kg} / \mathrm{ha}$ de $\mathrm{N}$ e $82,5 \mathrm{~kg} / \mathrm{ha}$ de $\mathrm{K}_{2} \mathrm{O}$ parcelado em quatro vezes.

A semeadura foi realizada em 08 de abril de 2011, em bandejas de poliestireno expandido, tipo 128/6, preenchidas com substrato comercial, sob ambiente protegido. As mudas foram transplantadas aos 22 dias após a semeadura, no espaçamento de $0,5 \times 0,3 \mathrm{~m}$, totalizando uma população de 66.000 plantas/ha.

Foi utilizada irrigação suplementar por aspersão com mangueira microfurada tipo Santeno, mantendo o solo próximo à capacidade de campo, monitorado com tensiomêtro. O controle das plantas invasoras foi realizado através do arranquio manual e com cobertura morta de grama esmeralda (Zoysia japonica), obtida por meio de poda da mesma em áreas gramadas da universidade.

A colheita das plantas foi realizada aos 76 dias após a semeadura. $\mathrm{Na}$ colheita, foi quantificado o número de plantas pendoadas por parcela. Foram colhidas seis plantas das linhas centrais de cada parcela, as quais foram levadas ao laboratório para a avaliação de produtividade $(\mathrm{PT})(\mathrm{t} / \mathrm{ha})$, peso $(\mathrm{PC})(\mathrm{g} /$ planta), diâmetro (DC) $(\mathrm{cm})$, e altura da "cabeça" (AC) (cm), comprimento do caule (CC) (cm), altura da planta (AP) (cm), número de folhas da saia (NFS), e compacidade (Cmp). Para esta característica foi adotada a escala utilizada para avaliação de compacidade da "cabeça" de alface americana, sendo $0=$ ausência total de "cabeça", 1= "cabeça" sem miolo definido, 2 = "cabeça" com miolo aparente e folhas periféricas soltas, $3=$ miolo definido e folhas iniciando compactação na periferia, $4=$ miolo definido e folhas da periferia compactas mas permitindo individualização visual e 5= miolo compacto e sem individualização visual das folhas periféricas. Essa metodologia foi adaptada de Antunes (2005) que trabalhou com alface.

Os dados obtidos foram submetidos à análise de variância e ao teste de separação de médias de Scott-Knott, a 5\% de probabilidade, utilizando o software Sistema para Análise e Separação de Médias em Experimentos Agrícolas (SASM-Agri) (Canteri et al., 2001).

\section{RESULTADOS E DISCUSSÃO}

Para a característica produtividade não houve diferença significativa entre as cultivares de couve-chinesa, obtendo-se médias variando de 1,35 a 1,64 kg/ planta. Para produção comercial e média de massa fresca da "cabeça" verificou-se, que as cultivares AF 66, Granat, Kioto e Kinjitsu, sem diferirem entre si, foram superiores às demais cultivares (Tabela 1).

A produtividade das cultivares utilizadas neste ensaio, de modo geral, foi considerada satisfatória, mesmo sendo cultivadas em condições tropicais de alta temperatura. Burt et al. (2006) afirmam que há potencial de produção de couve-chinesa de até 100 t/ha, porém boas produções comerciais variam de 50 a 70 t/ha.

Ito et al. (2006), ao realizarem estudo de competição de cultivares de couve-chinesa em Jaboticabal-SP, obtiveram massa fresca total variando de 2,25 a $3,26 \mathrm{~kg} /$ planta e comercial de 0,94 a 1,38 kg/planta, sendo superiores ao obtido neste experimento. Isso está relacionado à densidade populacional, que relaciona-se com a intensidade de competição entre plantas por espaço e luminosidade, sendo que, uma menor população de plantas por área (41.600 plantas/ha) pode favorecer o crescimento da planta. Porém, quando se compara a produtividade comercial por área, foi verificado que obtiveram-se produções equivalentes ou menores que as obtidas em Cáceres, mesmo sendo cultivados em condições climáticas consideradas desfavoráveis ao cultivo (Opeña et al., 1988; Filgueira, 2008).

Para a característica diâmetro da "cabeça" as cultivares podem ser divididas em quatro grupos, sendo o grupo com maiores diâmetros composto pelas cultivares Seijin, Kinjitsu e Kyoto, o segundo grupo AF66 e AF74 e o terceiro pela Granat e o último grupo pela Michihilli (Tabela 1). Para altura das plantas os resultados foram inversamente proporcionais ao diâmetro da "cabeça" onde, plantas com maior altura apresentaram menor diâmetro. Isso se dá pela característica genética dos materiais, sendo que há variação de formato (Opeña et al, 1988), podendo-se obter desde materiais cilíndricos do tipo Michihilli a mais ovalados, do tipo Wong Bok (Burt et $a l ., 2006)$. Essa variação de diâmetro e altura da "cabeça" também foi observada por Ito et al. (2006). Para o mercado local não se verifica a comercialização de materiais cilíndricos como Michihilli e Granat.

Quanto ao comprimento do caule foi observado que a cultivar Michihilli 
Tabela 1. Médias de produtividade (PT), produção da cabeça (PC), massa fresca da cabeça (MFC), diâmetro da cabeça (DC), altura da cabeça (AC), comprimento do caule (CC), compacidade (Cmp) e número de folhas da saia (NFS) em cultivares de couve-chinesa [average productivity (PT), production of heads (PC), head fresh weight (MFC), head diameter (DC), head height (AC), stem length (CC), compactness (Cmp) and number of leaves of the skirt (NFS) of Chinese cabbage cultivars]. Cáceres, UNEMAT, 2011.

\begin{tabular}{|c|c|c|c|c|c|c|c|c|}
\hline Cultivares & PT (t/ha) & $P C(t)$ & $\begin{array}{c}\text { MFC } \\
\text { (g/planta) }\end{array}$ & DC & $\begin{array}{l}\mathrm{AC} \\
(\mathrm{cm})\end{array}$ & $\mathrm{CC}$ & Cmp* & NFS \\
\hline Michihili & $89,36 \mathrm{a}$ & $38,54 \mathrm{~b}$ & $584,0 \mathrm{~b}$ & $5,5 \mathrm{~d}$ & $52,0 \mathrm{a}$ & $17,4 \mathrm{a}$ & $1,0 \mathrm{c}$ & $11,8 \mathrm{~b}$ \\
\hline Granat & $107,78 \mathrm{a}$ & $60,73 \mathrm{a}$ & $920,0 \mathrm{a}$ & $8,6 \mathrm{c}$ & $44,0 \mathrm{~b}$ & $12,4 \mathrm{~b}$ & $2,8 \mathrm{~b}$ & $12,8 \mathrm{~b}$ \\
\hline Seijin & 92,93 a & $50,13 \mathrm{~b}$ & $759,6 \mathrm{~b}$ & $13,3 \mathrm{a}$ & $30,2 \mathrm{~d}$ & $5,4 \mathrm{c}$ & $2,6 \mathrm{~b}$ & $15,2 \mathrm{a}$ \\
\hline Kinjitsu & $108,41 \mathrm{a}$ & $58,30 \mathrm{a}$ & $883,4 \mathrm{a}$ & $13,2 \mathrm{a}$ & $32,2 \mathrm{~d}$ & $5,4 \mathrm{c}$ & $2,9 \mathrm{~b}$ & $13,4 \mathrm{a}$ \\
\hline AF 66 & $98,51 \mathrm{a}$ & $60,96 \mathrm{a}$ & $923,6 \mathrm{a}$ & $10,3 \mathrm{~b}$ & $29,6 \mathrm{~d}$ & $4,6 \mathrm{c}$ & $4,3 \mathrm{a}$ & $11,2 \mathrm{~b}$ \\
\hline AF 74 & $79,98 \mathrm{a}$ & $45,42 \mathrm{~b}$ & $688,2 \mathrm{~b}$ & $11,3 \mathrm{~b}$ & $31,6 \mathrm{~d}$ & $4,2 \mathrm{c}$ & $2,4 \mathrm{~b}$ & $13,6 \mathrm{a}$ \\
\hline Kyoto & $106,87 \mathrm{a}$ & $59,06 \mathrm{a}$ & $894,8 \mathrm{a}$ & $12,6 \mathrm{a}$ & $35,8 \mathrm{c}$ & $7,8 \mathrm{c}$ & $2,2 \mathrm{~b}$ & $15,0 \mathrm{a}$ \\
\hline CV (\%) & 24,37 & 20,88 & 20,88 & 14,93 & 6,03 & 26,87 & 23,99 & 10,66 \\
\hline
\end{tabular}

Médias seguidas de mesma letra nas colunas não diferem entre si a 5\% de probabilidade pelo teste de Scott-Knott; *notas variando de 0 a 5 onde 0 = ausência total de "cabeça"; 1 = "cabeça" sem miolo definido; $2=$ "cabeça" com miolo aparente e folhas periféricas soltas; $3=$ miolo definido e folhas iniciando compactação na periferia; $4=$ miolo definido e folhas da periferia compactas mas permitindo individualização visual e 5= miolo compacto e sem individualização visual das folhas periféricas (means followed by same letters in column do not differ at $5 \%$ probability by Scott-Knott test; * grades varying from 0 to 5 where $0=$ no head formed; $1=$ head without defined heart; $2=$ head with apparent heart and periferic loose leaves; $3=$ defined heart and leaves beginning periferic compactation; $4=$ defined hearth and compact periferic leaves, permitting individual visualization and, $5=$ compact hearth withou individual visualization of periferic leaves).

apresentou a maior média $(17,4 \mathrm{~cm})$, característica essa não desejável para os consumidores e produtores, visto que, estas se encontravam com $9,1 \%$ das plantas florescidas no dia da colheita. Este florescimento foi observado por Silva et al. (2011) em cultivo em condições de inverno e relataram que essa cultivar não é recomendada para condições onde há oscilações entre baixas e altas temperaturas no período de cultivo, pois no período do cultivo as temperaturas médias foram de 16 a $28,3^{\circ} \mathrm{C}$, com variações de 8 a $40^{\circ} \mathrm{C}$.

Segundo Opeña et al. (1988) a temperatura ideal para a formação de "cabeça" varia de 15 a $20^{\circ} \mathrm{C}$. Maroto-Borrego (1995) ressalta que temperaturas inferiores a $12^{\circ} \mathrm{C}$ estimulam o florescimento. Feltrim et al. (2005), trabalhando com produção de couve-chinesa pak choi em diferentes épocas de cultivo, constaram que a interrupção do crescimento vegetativo e emissão da haste floral é determinada por baixas temperaturas, podendo ocorrer florescimento prematuro nas condições brasileiras de cultivo nas estações de outono, inverno e primavera, dependendo das condições da região, sendo a temperatura fator importante a ser considerado para se determinar a época de cultivo da couve-chinesa.
A cultivar Granat apresentou comprimento do caule intermediário, reduzindo a qualidade da "cabeça". As demais cultivares formaram um grupo com menor comprimento de caule o que pode conferir resistência ao pendoamento nas condições locais.

Uma das características de maior relevância é a compacidade da "cabeça", pois é desejado pelos consumidores uma boa formação de "cabeça", o que favorece a prevalência de folhas esbranquiçadas. Para os produtores esta é uma característica que reduz o volume de transporte e aumenta o peso por planta. Segundo Sousa (2000), para a alface americana, a boa formação de "cabeça" está vinculada ao fechamento compacto de suas folhas ao centro da planta, o que pode ser diretamente relacionado com a densidade, ou seja, quanto maior o peso e menor o volume, mais compactada será a "cabeça" e, portanto, de melhor qualidade.

Dentre as cultivares estudadas, a AF 66 teve melhor desempenho, seguida pelo grupo intermediário composto pelas cultivares Granat, Seijin, Kinjitisu, AF 74 e Kioto. O pior desempenho foi da cultivar Michihilli.

Já na característica de número de folhas da saia, destacaram-se as cultivares Seijin, Kinjtsu, AF 74 e Kyoto com maior número de folhas descartadas. Pode-se observar que a cultivar AF 66 obteve o melhor resultado dentre as cultivares, sendo material uniforme com boa adaptação para a cidade de Cáceres-MT, em condições de altas temperaturas.

Conclui-se, que em condições de temperaturas elevadas, há diferenças de natureza genética entre cultivares, destacando-se como promissoras pela massa fresca da "cabeça" e menor comprimento do caule as cultivares Kinjitsu, AF66 e Kyoto e, também pela maior compacidade a AF66.

\section{AGRADECIMENTOS}

Produção vinculada ao projeto de pesquisa "Aplicação e transferência de tecnologias na otimização de sistemas agrícolas sustentáveis", vinculado à sub-rede de estudos sociais, ambientais e de tecnologias para o sistema produtivo na região sudoeste mato-grossense - REDE ASA, financiada no âmbito do Edital MCT/CNPq/FNDCT/FAPs/ MEC/CAPES/PRO-CENTRO-OESTE $N^{\circ} 031 / 2010$ e ao projeto de Extensão processo $n^{\circ} 308283 / 2010$, financiado pela Fundação de Amparo à Pesquisa do Estado de Mato Grosso. 
Agradecemos também às empresas Sakata Seed Suldamerica, Agristar-Top Seed, Feltrin Sementes, Seminis Brasil e Isla Sementes pela indicação das cultivares e doação das sementes.

\section{REFERÊNCIAS}

ANTUNES CL. 2005. Fontes e modos de aplicação de potássio na alface americana (Lactuca sativa cv. Lucy Brown) em ambiente protegido. Botucatu: UNESP. 118p. (Tese doutorado).

BERNIER G; PÉRILLEUX C. 2004. A phsiological overview of the genetics of flowering time control. Plant Biotechnology Journal 3: 3-16.

BURT J; PHILliPS D; GATTER D. 2006. Growing chinese cabbage in western Australia. Australia: Department of Agriculture, 23p.

CANTERI MG; ALTHAUS RA; VIRGENS FILHO JS; GIGLIOTI EA; GODOY CV. 2001. SASM - Agri: Sistema para análise e separação de médias em experimentos agrícolas pelos métodos Scott-Knott, Tukey e Duncan. Revista Brasileira de Agrocomputação 1: 18-24.

EMBRAPA. EMPRESA BRASILEIRA DE PESQUISA AGROPECUÁRIA. 2006.
Sistema Brasileiro de Classificação de Solos. Brasília: Embrapa Produção de Informações. $306 \mathrm{p}$.

FELTRIM AL; REZENDE BLA; CECILIO FILHO AB. 2005. Produção de pak-choi em diferentes épocas de cultivo In: $45^{\circ}$ Congresso Brasileiro de Olericultura, Anais... Fortaleza: ABH. Disponivel em: <http:// www.a bhorticultura.com.br/biblioteca/ arquivos/Download/Biblioteca/44_287.pdf>. Acessado em 02 de junho de 2011.

FERREIRA WR; RANAL MA; FILGUEIRA FAR. 2002. Fertilizantes e espaçamento entre plantas na produtividade da couve-da-malásia. Horticultura brasileira 20: 635-640.

FILGUEIRA FAR. 2008. Novo manual de Olericultura: agrotecnologia moderna na produção e comercialização de hortaliças. Viçosa: UFV. 293p.

INMET. 2014. Disponível em: < http://www.inmet. gov.br/portal/index.php?r=bdmep/bdmep $>$ Acessado em 30 de março de 2014.

ITO LA; CARLO HCO; VARGAS PF; CASTOLDI RC; BRAZ LT. 2006. Produtividade e qualidade de cinco híbridos de couve-chinesa em campo aberto. In: $46^{\circ}$ Congresso Brasileiro de Olericultura, Anais... Goiânia: ABH. http://200.210.234.180/HORTA/Download/ Biblioteca/460248.pdf. Acessado em 02 de junho de 2011.

MAROTO-BORREGO JV. 1995. Horticultura herbácea especial. Madrid: Ediciones MundiPrensa. 611p.

NEVES SMAS; NUNES MCM; NEVES RJ. 2011. Caracterização das condições climáticas de Cáceres-MT Brasil, no período de 1971 a 2009: subsídio às atividades agropecuárias e turísticas municipais. Botetim Goiano de Geografia 31: 55-68.

OPEÑA RT; KUO CG; YOON JY. 1988. Breeding and seed production of chinese cabbage in the tropics and subtropics. Taiwan: Asian Vegetable Research and Development Centre (AVRDC). 92p.

SILVA MB; SEABRA JUNIOR S; RODRIGUES LFOS; OLIVEIRA RG; NOHAMA MTR; NUNES MCM. 2011. Incidência de pendoamento em couve-chinesa cultivadas sob campo aberto e telados. Horticultura Brasileira 29: S119-S125.

SOUZA LMA. 2000. Manejo da fertirrigação potássica na cultura da alface (Lactuca sativa L.) americana. Botucatu: UNESP. 63p. (Dissertação mestrado).

TRANI PE; PASSOS FAR; AZEVEDO FILHO JA. 1997. Recomendação de calagem e adubação para rúcula. In: RAIJ BV; CANTARELLA H; QUAGGIO JA; FURLANI AMC (eds). Recomendações de adubação e calagem para o Estado de São Paulo. Campinas: Instituto Agronômico e Fundação IAC. 285p. (Boletim técnico 100). 